\title{
Compressive Sensing Underwater Laser Serial Imaging System
}

Bing Ouyang*a ${ }^{\mathrm{a}}$ Fraser R. Dalgleish ${ }^{\mathrm{a}}$, Frank M. Caimi ${ }^{\mathrm{a}}$, Thomas E. Giddings ${ }^{\mathrm{b}}$, Joseph J. Shirron $^{\mathrm{b}}$, Anni K. Vuorenkoski ${ }^{\mathrm{a}}$, Walter Britton ${ }^{\mathrm{a}}$, Benjamin Metzger ${ }^{\mathrm{a}}$, Brian Ramos ${ }^{\mathrm{a}}$, Gero Nootz $^{\mathrm{a}}$

a. Florida Atlantic Univ./HBOI, 5600 US 1, Fort Pierce, FL, USA 34946

b. Metron Inc.1818 Library Street Suite 600, Reston, VA, USA 20190

\begin{abstract}
Compressive sensing (CS) theory has drawn great interest in recent years and has led to new image acquisition techniques in many different fields. This research investigates a CS based active underwater laser serial imaging system, which employs a Spatial Light Modulator (SLM) at the source. A multi-scale polarity flipping measurement matrix and a model-assisted image reconstruction concept are proposed to address limitations imposed by a scattering medium. These concepts are also applicable to CS based imaging in atmospheric environments characterized by fog, rain, or clouds. Simulation results compare the performance of the proposed technique with that of traditional laser line scan (LLS) sensors and other structured illumination based imagers. Experimental results from over-the-air and underwater tests are also presented. The potential for extending the proposed frame-based imaging technique to the traditional line-by-line scanning mode is discussed.
\end{abstract}

Keywords: laser imaging, underwater imaging, compressive sensing, scattering medium, spatial light modulation, laser line scan, serial imaging, electro-optical system

\section{BACKGROUND OF UNDERWATER IMAGERS}

Underwater imaging systems have been under continual development for use aboard undersea platforms such as towed-bodies, manned submersibles, Autonomous Underwater Vehicles (AUVs), 
and Remotely Operated Underwater Vehicles (ROVs). The imagery is used for various activities including scientific benthic survey, inspection of oil and gas infrastructures, and mine countermeasures operations. In many cases the optical imagers are used in conjunction with sonar. Under this operational concept, forward-looking and/or sidescan sonar is first employed to detect, classify and map potential targets and electro-optical imagery is acquired for target identification.

The most highly regarded extended range underwater laser imaging technique to date is the laser line scan (LLS) system [1-4]. The LLS imagers operate in serial mode. The scanning optics, electronics and mechanical components ensure that both the laser and telescopic receiver synchronously scan over a wide target region in line-by-line fashion. At each target element, a telescopic "bucket" photon detector such as a photomultiplier tube (PMT) records the photon flux from target reflections and generates output as an analog voltage. A high-speed analog to digital converter (ADC) digitizes the PMT output voltage to produce an uncompressed image of the scene. The image can then be compressed using a codec such as JPEG.

With one scheme commonly referred to as near-monostatic synchronous LLS (Figure 1a), where the illuminator and receiver are co-located on the same platform, the imager scans one line of the target scene at a time, and relies on the forward motion of the platform to complete the image of the entire scene in a whisk-broom fashion. Alternatively, the concept of a Time Varying Intensity (TVI) system, originally demonstrated in the 1970s [5], has led to Distributed Laser Serial Imaging (DLSI), which is a non-synchronous scanning technique. With DLSI, the laser transmitter and optical receiver are distributed amongst multiple platforms with the objective of minimizing volume scattering effects using large separation distances between the transmitter and receiver (Figure 1b). DLSI systems have produced results beyond 30 meters in turbid coastal waters [6]. 


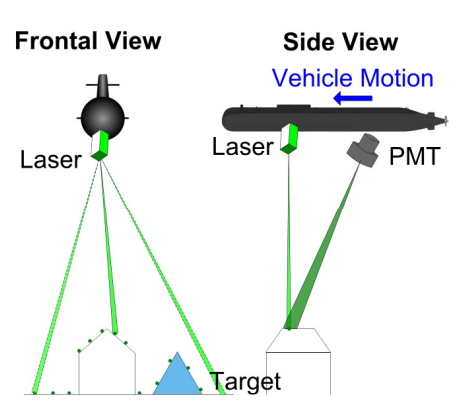

(a) Synchronous serial imaging

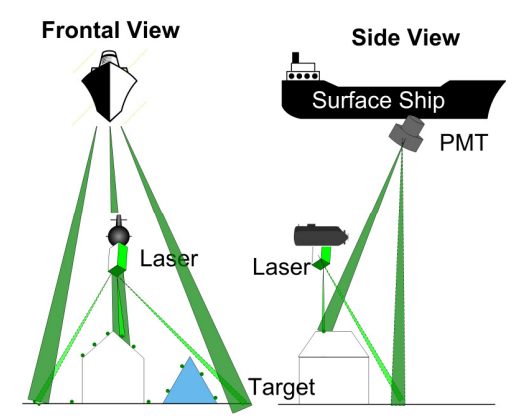

(b) Distributed serial imaging

Figure 1. Illustration of the operation of two types of underwater laser serial imaging systems.

For both near-monostatic and distributed laser serial imaging systems, opto-mechanical components such as a high precision prisms, galvanometric scanners and/or aperture assemblies are needed to ensure proper operation. Such components lead to high production and maintenance costs, consume a significant portion of the system size, weight and power (SWAP) budget, and the many moving parts can reduce system reliability. The optical and mechanical parts also lead to bulky system packaging, where extended range undersea laser imagers must be compact to be compatible with current and future classes of man-portable AUVs.

In pursuit of more compact, lower cost, and highly reliable system concepts, a structured illumination and ranged gated camera (SIRGC) technique was recently described [7]. Digital Mirror Device (DMD) based confocal optics were used to spatially modulate a laser pulse into multiple beams that were separated by a pre-defined distance at the target plane. Such separation ensured that there was less overlap between beams after spreading in turbid water. The target reflection was modulated by the DMD and focused onto a range-gated camera via the same confocal optics. Finally, an image synthesis process constructs the uncompressed image using all of the sub-images (Figure 2b). The SIRGC technique shares the same image acquisition paradigm as the aforementioned laser serial imaging systems in that the raw uncompressed data is first acquired and then a codec such as JPEG is used to compress the data before storage. 


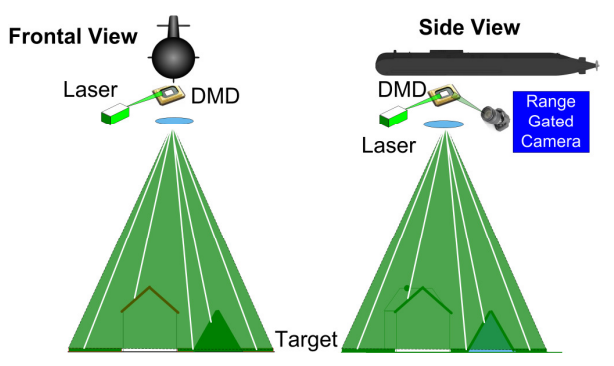

(a) Illustration of the operation mode

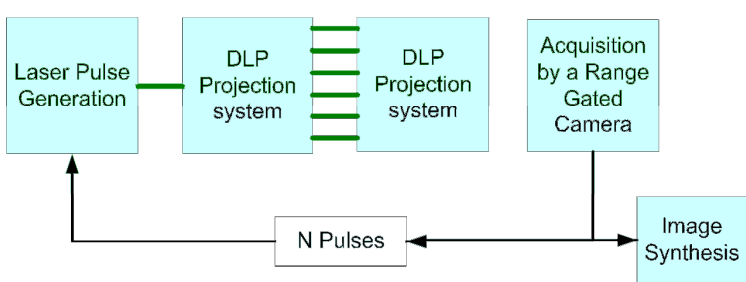

(b) Process flow [7]

Figure 2. Structured illumination based imager (SIRGC) [7].

In recent years, compressive sensing theory has drawn considerable interest in connection with various signal acquisition applications. However, there has not been any reported development of CS based underwater laser imagers. This work investigates the feasibility of such a concept. As a first attempt, only imaging of a static scene is considered.

\section{COMPRESSIVE IMAGING}

\subsection{Fundamentals of Compressive Sensing}

Compressive sensing (CS) is a framework for the simultaneous sampling and compression of sparse (therefore compressible) signals using incomplete linear measurements [8-14]. We consider a discretely sampled signal $X=\{x(n), n=1,2, \ldots, N\}$. If there exists a sparsifying basis $\Psi=$ $\left\{\psi_{1}, \psi_{2}, \ldots, \psi_{N}\right\}$ for the $N$-dimensional signal $X$, such that $X=\Psi \alpha$ and the $N \times 1$ vector $\alpha$ contains only $K \ll N$ non-zero entries, and then $X$ is referred to as a $K$-sparse signal. CS theory states that a $K$-sparse signal $X$ can be recovered with overwhelming probability using more than $M=O(K \log N)$ incoherent linear measurements: $y=\Phi X=\Phi \Psi \alpha$, where $y$ is an $M \times 1$ vector and $\Phi$ is an $M \times N$ measurement matrix that is incoherent with the sparsifying basis $\Psi$ (i.e., the maximum magnitude of the elements of $\Phi \Psi$ is small [6]). The recovery can be achieved when $\Phi$ satisfies the Restricted Isometry Property (RIP) $[8,14]$. Random bases such as Gaussian, Bernoulli, or scrambled block Hadamard Ensemble $[12,15]$ are some common choices for $\Phi$ that satisfy the RIP constraint. A 
sparsifying basis $\Psi$ exists for many signal types. For example, natural images are generally sparse in the Fourier, DCT or wavelet domain, a property exploited in the compression standards such as JPEG. The vector $\alpha$ (therefore $X$ ) can be recovered from the measurements $y$ by solving an $l_{1}$-minimization problem [10]:

$$
\begin{aligned}
& \alpha^{*}=\arg \min \|a\|_{1} \\
& \text { subject to } y=\Phi \Psi a
\end{aligned}
$$

where $\|a\|_{1}=\sum_{i=1}^{N}\left|a_{i}\right|$ is the $l_{1}$-norm of $a$.

The application of CS theory to image and video acquisition, or Compressive Imaging (CI), has drawn extensive interest. In addition to the aforementioned sparsifying bases, the image gradient sparsity can be exploited via minimization of the image total variation (TV), which is the sum of the magnitudes of discrete gradients $D_{i j}(X)$ at every point in the digital image $X$ :

$$
T V(X)=\sum_{i j}\left|D_{i j}(X)\right|=\sum_{i j} \sqrt{D_{h ; i j}^{2}(X)+D_{v ; i j}^{2}(X)}
$$

where $D_{i j}(X)$ is defined as

$$
D_{i j}(X)=\left(\begin{array}{c}
D_{h ; i j}(X) \\
D_{v ; i j}(X)
\end{array}\right),
$$

with $D_{h ; i j}=x_{i+1, j}-x_{i, j}$ and $D_{v ; i j}=x_{i, j+1}-x_{i, j}$. TV minimization with quadratic constraints

$$
\begin{aligned}
& X^{*}=\arg \min T V(X) \\
& \text { subject to }\|A X-y\|_{2} \leq \epsilon
\end{aligned}
$$

has been shown to provide estimates with better visual quality than $l_{1}$-optimization when recovering images from observations subject to additive Gaussian noise [16-19].

\subsection{Compressive Imaging Applications}

The Rice one-pixel camera was one of the earlier successful implementations of Compressive Imaging [13-14]. The core of the design was a binary spatial light modulator (SLM) based on a DMD. The DMD consists of millions of electrostatic actuated micro-mirrors that can be individually controlled to reflect light into one of two directions to modulate the incoming light source in binary 
mode. While widely used as display devices in DLP $^{\circledR}$ TVs or data projectors, the DMD was also used as an SLM device due to its high contrast ratio and fast switching speed [20].

For the Rice one-pixel camera project, a light emitting diode (LED) illuminated the target; the target reflection was focused onto a DMD. The $m^{\text {th }}$ random pattern from the measurement matrices $\left\{A_{m}, m=1,2, \ldots, M\right\}$ was loaded onto the DMD to modulate the target reflection. The modulated light was then focused onto a photodiode whose output was integrated to provide the $m^{\text {th }}$ scalar measurement in the vector $\left\{y_{m}, m=1,2, \ldots, M\right\}$. An optimization process such as basis pursuit then reconstructed the image $X$ using the $M$ binary measurement matrices $\left\{A_{m}\right\}$ and the $M$ measurements $\left\{y_{m}\right\}$. While no detail was provided, an active illumination based one-pixel camera was also alluded to in [14].

CS theory has been adopted in other imaging applications as well. For example, Lustig et al. [21] adopted CS for rapid magnetic resonance imaging (MRI), another early CS imaging application; Ma [22] proposed CS based single-pixel multiple times (SPMT) imaging for aerial remote sensing and Baraniuk \& Steeghs [23] also applied CS to Radar imaging.

\section{CS UNDERWATER LASER IMAGER}

\subsection{Basic System Framework}

The proposed CS underwater laser imager is an active serial imaging system. It can therefore be considered as a hybrid of the Rice one-pixel camera, structured illumination, and the LLS system (Figure 3). During the sensing process, the illuminating laser is focused onto the DMD loaded with the binary illumination patterns $\left\{A_{m}\right\}$, which are projected onto the target surface in a serial mode. At the receiver, as with the LLS system, a PMT measures the total photon flux in response to the modulation of each pattern with the target plane. The PMT output voltages are integrated to generate the measurements $\left\{y_{m}\right\}$. The illumination patterns $\left\{A_{m}\right\}$ and the corresponding measurements 
$\left\{y_{m}\right\}$ are provided as inputs to an optimization process that reconstructs the image. The transmitter and receiver can be co-located, like in the near mono-static LLS system, or on different platforms like the DLSI.

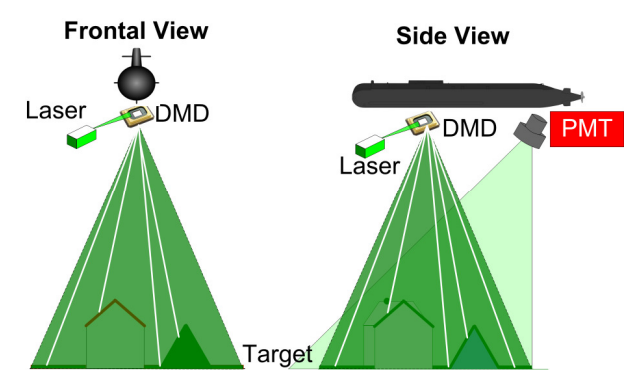

Figure 3. Illustration of CS underwater imaging system.

For the underwater system, the measurement matrices $\left\{\Phi_{m}\right\}$ are, essentially, the illumination patterns propagated to the target plane through the turbid underwater environment. So, to discuss the system implementation in detail it is important to understand the impact of light propagation in the underwater environment on the sensing process (i.e., the modulation of the measurement matrices with the target scene reflectance pattern), which is the critical component in any CS application.

\subsection{Impact of the Underwater Environment on the Sensing Process}

Compared to the over-the-air scenarios, the main challenge for underwater CS imagers is the pronounced beam spreading and attenuation due to the propagation of light through a scattering and absorbing medium such as ocean water. The effects of solar irradiance/ambient light are omitted in the analysis presented herein.

When a binary pattern of highly collimated light is emitted from the transmitter, the light will diffuse and attenuate as it propagates to the target plane. The degree of spreading/attenuation is determined by the laser beam divergence, target range, and the inherent optical properties (IOPs) of the water, such as the beam attenuation coefficient $(c)$, the absorption coefficient $(a)$, and the scattering coefficients $(b)$, where $c=a+b$. The target scene reflection is given by the product of 
the incident light pattern and the target surface reflectivity pattern $X$. The reflected light will then undergo additional spreading and attenuation as it propagates to the PMT receiver. The photon flux corresponding to the target scene reflection that enters the receiver is a function of the PMT location and orientation, its sensitivity pattern, and the IOPs. In addition, the (non-target information bearing) photon flux due to volume backscatter in the water will also contribute to the measured signal.

Assuming a wide receiver aperture and Lambertian reflection at the scene, the total photon flux corresponding to the $m^{\text {th }}$ measurement $y_{m}$ can be represented by the equation ( $*$ denotes convolution and $\circ$ represents the Hadamard point-by-point product)

$$
y_{m}=S_{R} \Omega_{\mathrm{R}} \frac{E}{N_{1, m}} \sum_{i, j}\left[\alpha_{T R}\left(A_{m}(i, j) * B S F_{I T}(i, j)\right) \circ X(i, j)+\hat{\beta}_{m} A_{m}(i, j)\right]
$$

where $A_{m}$ is the $m^{\text {th }}$ binary illumination pattern (i.e., consisting of "on" (1) and "off" (0) pixels) emitted from the transmitter and projected down to the scene (without scattering or absorption); $N_{1, m}$ is the number of "on" pixels in the pattern (i.e., where $A_{m}(i, j)=1$ ); $S_{R}$ and $\Omega_{R}$ are the receiver surface area and solid angle, respectively, which are both constant; $E$ is the total energy output of the illuminator for each projected pattern; $B S F_{I T}$ is the beam spread function from the illuminator to the target (see [24] and references therein); and, $\alpha_{T R}$ is a constant representing the diffuse attenuation from the target to the receiver. Making the reasonable assumption that the volume backscatter contribution from each beam $(\hat{\beta})$ in the illumination pattern is the same; the total volume backscatter component of the measurement is proportional to the number of "on" pixels in the pattern $N_{1, m}$.

The essence of the sensing process is to "imprint" the target scene information on the measurements through the modulation of the projected light pattern according to the spatially varying reflectivity of the scene. In this regard, it is beneficial to rewrite Eq. (5) as: 


$$
y_{m}=S_{R} \Omega_{\mathrm{R}} E\left(\frac{\alpha_{T R}}{N_{1, m}} \sum_{i, j}\left[\Phi_{m}(i, j) \circ X(i, j)\right]+\hat{\beta}_{m}\right)
$$

In Eq. (6), $\Phi_{m}=A_{m} * B S F_{I T}$ is the measurement pattern on the target plane (i.e., the original binary pattern after propagating from the illuminator to the target plane through a scattering and absorbing medium), $\Phi_{m} \circ X$ is the resulting "imprinted" pattern due to the modulation of the measurement pattern by the target scene reflectance pattern, and $y_{m}$ is the corresponding total reflected photon flux.

Equation (6) reveals some important factors affecting the underwater CS laser imager design. First, if the binary illumination pattern $A_{m}$ is dense (Figure 4a), the details in the corresponding measurement matrix $\Phi_{m}$ will be lost due to the low-pass filtering effect of $B S F_{I T}$ (Figure $4 \mathrm{~b}$ ). We also emphasize that the original binary patterns $A_{m}$ that enter the water are not the "sensing" patterns that illuminate the target plane; the modulation occurs between the target scene reflectance pattern and $\Phi_{m}$ instead of $A_{m}$. The impact of propagation from the target to the receiver on the total photon flux is essentially diffuse attenuation that can be represented by an attenuation factor $\alpha_{T R}$, which remains constant for all the patterns in the sensing process. Based on these discussions, the measurement matrix design to overcome these challenges is presented.

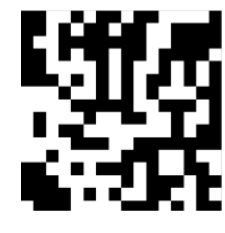

(a) Original $A_{m}$

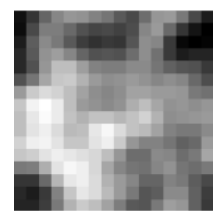

(b) $\Phi_{m}$ at 5 Attn. Lengths

Figure 4. Binary illumination pattern and measurement matrix in turbid water.

\subsection{Measurement Matrix Design}

Essentially, there are three unique aspects of the proposed measurement matrix design: 


\section{Model Assisted Reconstruction}

As described in Sections 3.1 and 3.2, the sensing process is characterized by the modulation of the target plane reflectance pattern $X$, with the sensing patterns $\left\{\Phi_{m}\right\}=\left\{A_{m}\right\} * B S F_{I T}$, whereas the propagation from the target to the receiver can be represented by the diffuse attenuation factor $\alpha_{T R}$, which introduces no new "information". With this in mind, $\left\{\Phi_{m}\right\}$ will be used in the image reconstruction process. To predict $\left\{\Phi_{m}\right\}$, the EODES radiative transfer models [24] have been extended in a collaborative effort between HBOI and Metron. EODES physical models can predict the image under given environmental parameters and system configuration. While the EODES models are typically used for at-sea performance prediction and to correlate with experiment data for theoretical analysis, this research proposes to incorporate results from model predictions into the actual image reconstruction process for underwater CS imagers.

\section{$\underline{\text { Multi-scaled Binary Pattern }}$}

To mitigate the beam spreading due to forward scattering in the water, a multi-scale binary dither pattern (Figure 5) will be adopted instead of using a dense binary pattern. The dither pattern is divided into small $N_{b} \times N_{b}$ blocks. Only one "on" pixel will be present within each block and the location of this pixel within the block will be determined by a discrete random variable with uniform probability mass, $1 / N_{b}^{2}$. The polarity of each block will be determined by an independent Bernoulli random variable where the polarity is equally likely to be " 1 " or " 0 " (i.e., on or off).

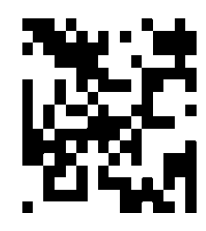

(a) Top level

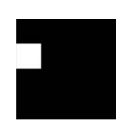

(b) One block

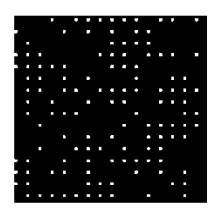

(c) Overall matrix

Figure 5. Multi-scale measurement matrix. 


\section{Bipolar Matrices and Measurements via Polarity Flipping}

During image reconstruction, the positive-valued (model predicted) measurement matrices $\left\{\Phi_{m}\right\}$ and the raw measurements $\left\{y_{m}\right\}$ will be converted to bipolar matrices $\left\{\Phi_{m}^{ \pm}\right\}$and vectors $\left\{y_{m}^{ \pm}\right\}$:

$$
\begin{aligned}
& \left\{\Phi_{m}^{ \pm}\right\}=C\left(\left\{\Phi_{m}\right\}-\operatorname{bias}\left(A_{m}\right)\right) \\
& \left\{y_{m}^{ \pm}\right\}=y_{m}-\operatorname{bias}\left(y_{m}\right)
\end{aligned}
$$

where \pm indicates that both positive and negative values are possible, and $C$ is a normalization factor. In many implementations, as with the Rice single pixel camera, the bias is the DC shift across all patterns and it can be pre-measured and subtracted to create the bipolar patterns/measurements. However, such is not the case with the proposed design, where the backscatter level varies with the different patterns (i.e., the backscatter level is sensed together with the target reflection). Furthermore, $\left\{\Phi_{m}\right\}$ is not a binary signal due to the low-pass filtering effect (Figure 6).

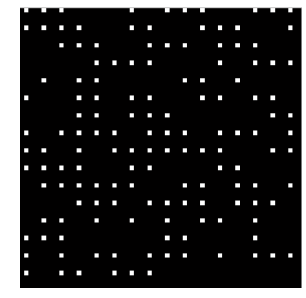

(a) $A_{m}$

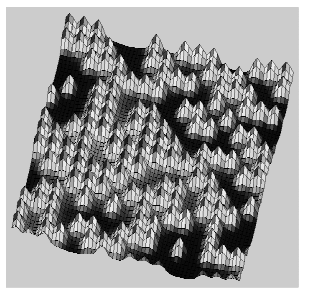

(b) $\Phi_{m}$

Figure 6. Original binary pattern $A_{m}$ and the model predicted measurement matrix $\Phi_{m}$.

One possible option is to set the bias to the mean of the measurements and measurement matrices; but the resulting measurement matrices will not be symmetric, and this approach does not consider the additional variance in background level due to changing ambient light conditions in an operational scenario. As an alternative, a polarity flipping technique is adopted. To implement polarity flipping, each dither pattern is loaded twice, first with the mirrors "on" (corresponding to a digital "1") only in the blocks originally chosen to be "on", producing the pattern $\Phi_{m}^{+}$, and then with mirrors "on" only in the blocks originally selected to be "off”, producing $\Phi_{m}^{-}$. The relations 


$$
\begin{aligned}
& \Phi_{m}^{B}=C\left(\Phi_{m}^{+}-\Phi_{m}^{-}\right) \\
& y_{m}^{B}=y_{m}^{+}-y_{m}^{-}
\end{aligned}
$$

where $y_{m}^{+}$and $y_{m}^{-}$are the measurements corresponding to $\Phi_{m}^{+}$and $\Phi_{m}^{-}$, represent the sensing process when polarity flipping is employed.

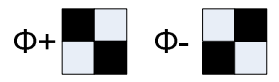

(a) Mirror polarity flipping

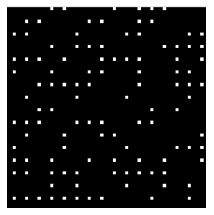

(b) $A_{m}^{+}$

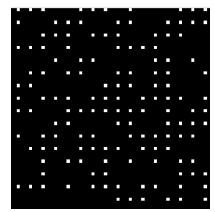

(c) $A_{m}^{-}$

Figure 7. Mirror polarity flipping to reduce volume backscattering.

\subsection{Overall System Design}

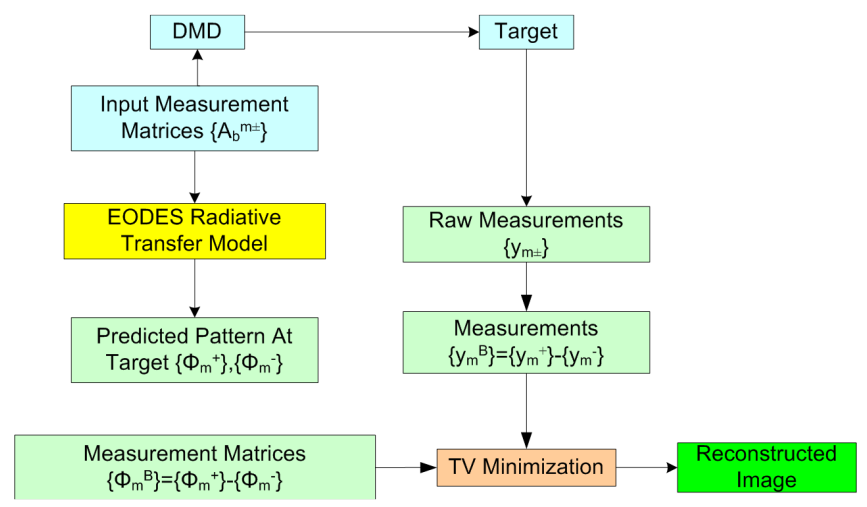

Figure 8. Compressive sensing based underwater laser imager system flow chart.

The overall system is summarized in the flowchart of Figure 8. A series of pre-generated binary illumination patterns $\left\{A_{m}\right\}$ are loaded onto an SLM device such as the DMD to spatially modulate the laser source that illuminates the target plane. The same binary matrices are also submitted to a radiative transfer model such as EODES to predict the measurement matrices $\left\{\Phi_{m}\right\}$ at the target plane. A PMT type "bucket" photon collector records the total optical return signal. The difference between polarity-flipped pairs of measurement matrices (predicted with opposite polarity binary patterns) constitutes $\left\{\Phi_{m}^{B}\right\}$, which is one input to the reconstruction process; and the difference 
between their corresponding PMT measurements constitutes $\left\{y_{m}^{B}\right\}$, the other input to the reconstruction process. In the current the implementation, TV minimization under a quadratic constraint is adopted for the image reconstruction process.

A rigorous proof that the proposed measurement matrix design satisfies the RIP constraint will be presented in future work. However, one specific case where the "on" pixel $(i$ ', $j$ ') is fixed at the center of a block $(u, v)$ : $\left(u N_{b}+N_{b} / 2, v N_{b}+N_{b} / 2\right)$ (assuming a square block of dimension $\left.N_{b}\right)$ is briefly discussed here. In this case, the "on"/“off” blocks are independent Bernoulli random variables with equal probability from the set $\{-1,1\}$. For the purpose of illustration, we approximate the $B S F_{I T}$ as a Gaussian kernel: $B S F_{I T}(i, j)=\rho e^{-\beta\left(i^{2}+j^{2}\right)}$. Therefore, a pixel $(i, j)$ in the sensing pattern $\left\{\Phi_{m}^{B}\right\}$ can be expressed as:

$$
\Phi_{m}^{B}(i, j)=\rho \sum_{u, v=0}^{U, V} \mathrm{~A}_{m}^{B}(u, v) e^{-\beta\left[\left(i-\left(u N_{b}+N_{b} / 2\right)^{2}+\left(j-\left(v+N_{b} / 2\right)\right)^{2}\right]\right.}=\rho \sum_{u, v=0}^{U, V} \mathrm{~A}_{m}^{B}(u, v) e^{-\beta r^{2}}
$$

where $A_{m}^{B}=A_{m}^{+}-A_{m}^{-}$, and UV is the total number of blocks in the image. From Eq. (9), the contribution of a neighboring block $(u, v)$ to pixel $(i, j)$ is determined by the Bernoulli random variables $A_{m}^{B}$ with value from the set $\{1,-1\}$, and $r=\sqrt{\left(i-\left(u N_{b}+N_{b} / 2\right)^{2}+\left(j-\left(v+N_{b} / 2\right)\right)^{2}\right.}$, the distance between the two pixels. Applying a normalization factor $\left(\rho \sum_{u^{\prime}, v^{\prime}=0}^{U, V} e^{-\beta{r^{\prime}}^{2}} \sqrt{U V}\right)^{-1}$, then Eq. (910) can be rewritten as:

$$
\hat{\Phi}_{m}^{B}(i, j)=\frac{1}{\rho \sum_{u^{\prime}, v^{\prime}=0}^{U, V} e^{-\beta r^{\prime 2}} \sqrt{U V}}\left(\rho \sum_{u, v=0}^{U, V} A_{m}^{B}(u, v) e^{-\beta r^{2}}\right)=\sum_{u, v=0}^{U, V} \frac{1}{\sqrt{U V}} A_{m}^{B}(u, v) \frac{e^{-\beta r^{2}}}{\sum_{u^{\prime}, v^{\prime}=0}^{U, V} e^{-\beta r^{\prime 2}}}=\sum_{u, v=0}^{U, V} S(r)
$$

The $\{S\}$ in Eq. (10) can alternatively be regarded as discrete independent random variables with the probability distribution: 


$$
S(r)=\left\{\begin{array}{l}
\frac{1}{\sqrt{U V}}, \quad \hat{p}=\frac{1}{2} \frac{e^{-\beta r^{2}}}{\sum_{u^{\prime}, v^{\prime}=0}^{U, V} e^{-\beta r^{2}}} \\
-\frac{1}{\sqrt{U V}}, \hat{p}=\frac{1}{2} \frac{e^{-\beta r^{2}}}{\sum_{u^{\prime}, v^{\prime}=0}^{U, V} e^{-\beta r^{2}}}
\end{array}\right.
$$

It can be easily derived that $\{S\}$ has mean $E[S]=0$; and variance: $E\left[S^{2}\right]=1 / U V$, where $E[\cdot]$ indicates the expectation. Because $\{S\}$ are iid random variables, based on the central limit theorem, $\left\{\widehat{\Phi}_{m}^{B}\right\}$ are Gaussian-like random variables. In this case, $\left\{\widehat{\Phi}_{m}^{B}\right\}$ satisfies the Restricted Isometry Property (RIP) from the results in [12] and [17].

\subsection{Advantages of the CS based Underwater Laser Imager}

As with the SIRGC design, a commercially available solid-state SLM device such as a DMD replaces the bulky mechanical and optical components used in the conventional LLS. This helps to improve the system reliability and compactness. Furthermore, the compressing-during-sampling paradigm requires lower-speed electronics, which in turn improves system noise performance and reduces production costs. Because the CS imager uses the "bucket" photon collector such as a PMT, like the LLS system except with a much wider instantaneous FOV, the photon efficiency should be better than that of the structured illumination based system.

One interesting aspect of the proposed design is that the receiver will need both the measurements as well as the measurement matrices to reconstruct the image. In this sense, CS based imaging can be considered an encrypted communication channel. With the distributed imaging and communication arrangement [6], where the illuminators and receivers are on different platforms, this attribute is highly desirable since both compression and encryption can be realized without additional hardware. Also, because each measurement is independent of all others, the loss of certain 
measurements can be regarded as image reconstruction with increased compression ratio as long as the indices of these lost measurements are known. The encryption property of CS has been studied in connection with other applications, such as in [25] and [26].

When one considers the existing components used in the LLS systems, the proposed CS system only replaces the scanning mechanism with a solid-state SLM device. The laser and PMT developed for conventional LLS can be readily used in a CS based imager. Additionally, effective volume backscatter reduction techniques developed for the LLS system, such as the pulsed laser and rangegated receiver and the modulated pulsed laser, remain applicable to the CS based imaging system.

In addition to the simplified illuminator design, which is highly desirable in distributed underwater imaging applications, the CS based imager shares another feature with the multi-static underwater LLS system in that it does not require direct line-of-sight between the target plane and receiver in turbid water $[6,27]$. This is one of the major motivations for adopting an SLM based illuminator over the receiver based approach as demonstrated in the Rice one-pixel camera.

\subsection{Potential Application Scenario for CS based Underwater Laser Imager}

One potential application of the CS underwater laser imager is as a long-range, high-resolution underwater imaging system that integrates the CS imager with an acoustic communications (ACOM) link (Figure 9). At the front end, the illuminator consists of the laser and an SLM device such as a DMD. The pre-stored binary illumination patterns are loaded onto the DMD to modulate the laser. The PMT at a local receiver (either co-located on the same platform as the illuminator or on a different platform) records the total target reflection. Its output is digitized and fed into an acoustic transducer to transmit the data to the remote control center. The IOPs of the water column and the imaging system parameters are also streamed with the measurement data. At the control center, the same binary illumination patterns, together with the IOP data and imaging system parameters, are 
fed into a computationally efficient radiative transfer model like EODES to compute the measurement matrices. These predicted measurement matrices are then used in conjunction with the transmitted measurement data to reconstruct the target image.

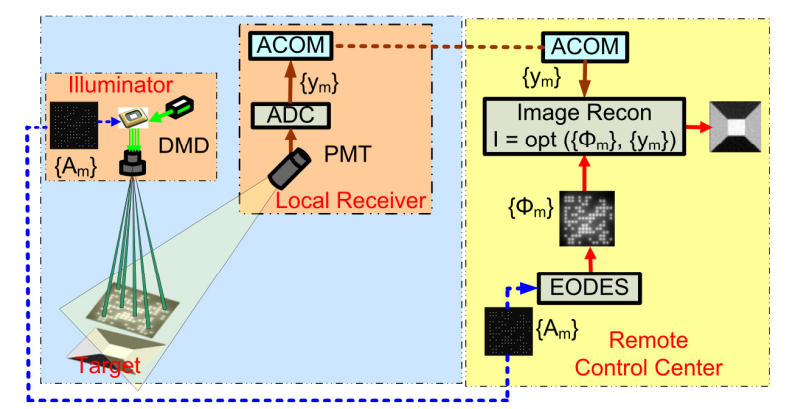

Figure 9. Long-range, high-resolution imaging using CS imager.

As described in section 3.6, the communications link in this integrated system is intrinsically encrypted and compressed without requiring additional hardware. The system takes advantages of the high-resolution reflectance imaging capability offered by the CS imager as well as the extended communication range achievable via the ACOM link. For example, to reconstruct an image with $256 \times 256$ pixels at a 10:1 compression ratio requires about 6000 measurements. If an ADC with 12-bit resolution is used, then to transmit/reconstruct an image within 1 second would require a $70 \mathrm{kbps}$ communications link. Such a link with a range of several kilometers is achievable using underwater acoustic communication systems [28].

\section{SIMULATION AND EXPERIMENT}

\subsection{Description of Simulation Environment}

\section{Development of the Simulation Environment}

The core of the simulation environment was the EODES radiative transfer model developed for near mono-static underwater electro-optical systems [24]. The EODES model suite consist of both an image simulation tool (EODES-I) and a temporal simulation tool (EODES-T), to study the timedependent propagation of a laser pulse in the underwater environment. EODES-I relies on the small- 
angle scattering approximation to achieve semi-analytical models that enable fast simulation speeds. EODES-T simulations can be conducted with small-angle scattering models or the Monte Carlo method, the latter providing more accurate results at the expense of longer execution times.

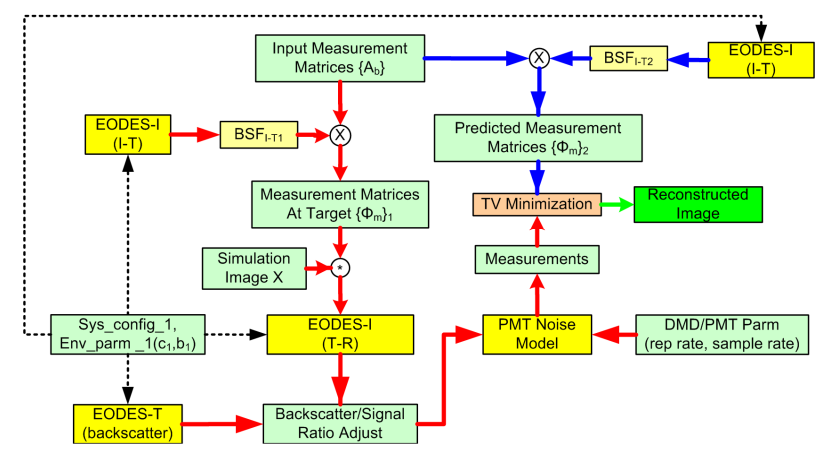

Figure 10. Simulation flow.

Both EODES-I and EODES-T were used in the current CS study. The simulation environment underwent iterative enhancements to improve the fidelity of the simulation results. Figure 10 illustrates the current overall simulation environment. Different beam attenuation $(c)$ and/or scattering $(b)$ coefficients can be specified for the simulation path (red arrows) and the modelprediction path (blue arrows) when predicting the measurement matrices using the EODES-I model. Furthermore, simulated PMT device noise can be added to the measurements to capture the effects of shot noise and random variations in PMT gain [29-30]. One critical issue, especially when evaluating system performance under PMT noise corruption, is obtaining the signal intensity and the ratio between backscatter to signal level. For this purpose, the same system and environmental parameters are used as input to the EODES-T temporal model to derive the backscatter and signal levels using the Monte Carlo technique.

$\underline{\text { PMT Noise Model }}$

A crucial component of the simulation is the noise model for PMT devices [29-30]. Instead of assuming simple Poisson counting statistics, the PMT shot noise is modeled as a compound Poisson 
stochastic process, where noise is added to a clean signal on a per-sample basis. The random sequence of the detector output stream is modeled as a multivariate Gaussian distribution where $p$ adjacent samples are correlated. Furthermore, the model also takes into consideration the impact on the noise performance of the various PMT parameters such as bandwidth and sampling rate. Random PMT gain $(G)$ fluctuations, as measured by the noise factor $N F=\left\langle G^{2}\right\rangle /\langle G\rangle^{2}$, were also incorporated into the model.

\section{$\underline{\text { Simulation Setup }}$}

Simulations were conducted for four different technologies: CS, Pulsed LLS (PLLS), Continuous Wave (CW) LLS, and SIRGC. A near-monostatic configuration was adopted for all simulations. The laser and receiver (PMT or range gated camera) separation was set to $0.4 m$ and the laser divergence angle was $2 \mathrm{mrad}$. The target scene was $1.2 \mathrm{~m} \times 1.2 \mathrm{~m}$ and was located $7 \mathrm{~m}$ away from the illuminatorreceiver assembly. The resolution of the image and the sensing patterns was $64 \times 64$ pixels. Unless stated otherwise, average laser power was $4 \mathrm{~W}$ and the DMD refresh rate was $4 \mathrm{KHz}$. For CS image reconstruction, 1024 measurements were used, resulting in a compression ratio of 0.25 . (Note: due to polarity flipping 2048 raw measurements were acquired). In the related simulations of other technologies (PLLS, CW LLS and SIRGC), the same laser power was used. For PLLS the laser pulse repetition rate $(\tau)$ was $4 \mathrm{KHz}$. For the SIRGC, the repetition rate was set to $100 \mathrm{~Hz}$. In PLLS and SIRGC simulations, a $3 n s$ laser pulse was used and perfect range gating (i.e., no backscatter reached the PMT) was assumed. In the CS and the SIRGC simulations, since the illumination was frame based, the input power for each pixel element was set to $P_{\text {pixel }}=P_{\text {total }} /\left(N^{2} \tau\right)$, where $N^{2}$ is the pixel count, to match the power levels in the PLLS and CW LLS simulations.

The receiver apertures were set to $15 \mathrm{mrad}$ for the PLLS and CW LLS simulations, $90 \mathrm{mrad}$ for the CS simulations, $0.5 \mathrm{rad}$ for the SIRGC simulations (per [7]). In the CS simulations, the pixel 
spacing (i.e., scale) was set to four pixels. For the SIRGC simulations the spacing was set to 10 pixels (this approximately matched the case of 25 -pixel spacing for $256 \times 256$ pixel resolution in the simulations in [7]), hence 100 sub-frames were needed for the image synthesis. Unless specified otherwise, the same measurement process outlined in Figure 10 was applied. A noise factor $N F=1.2$ was assumed throughout the simulations. For the SIRGC simulations, the same PMT noise model was adopted, but with each sample taken to be uncorrelated.

To evaluate the simulation image quality, a well-known image quality metric, the structural similarity index metric (SSIM), was adopted. Please refer to [31] for more detail on SSIM.

\subsection{Simulation Results}

Target range is specified in terms of beam attenuation lengths, where one beam attenuation length (AL) equals the reciprocal of the beam attenuation coefficient: $1 \mathrm{AL}=1 / c$ meters. The measurement matrices at different beam attenuation lengths are shown in Figure 11. While the beam spreading increased with higher turbidity, the patterns maintain discernible separation.

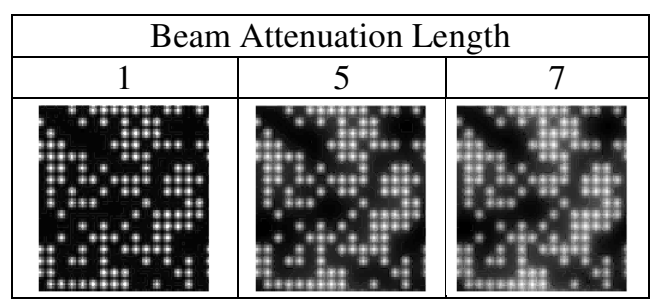

Figure 11. Multi-scale measurement matrix at different turbidities.

The comparison of model-assisted reconstructions with and without polarity flipping, as well as reconstructions using binary matrices (with polarity flipping), at 7 AL were evaluated using the Star Chart (Figure 12). When no polarity flipping was used, the measurements and measurement matrices were converted to bipolar signals by subtracting the mean of all measurements/measurement matrices and normalizing to unit amplitude. When there was no noise added, better contrast and resolution were achieved using model predicted measurement matrices as opposed to using the 
binary illumination patterns. There was no significant difference when polarity flipping was employed in the absence of noise. However, when noise was added, better image resolution seemed to result when polarity flipping was engaged.

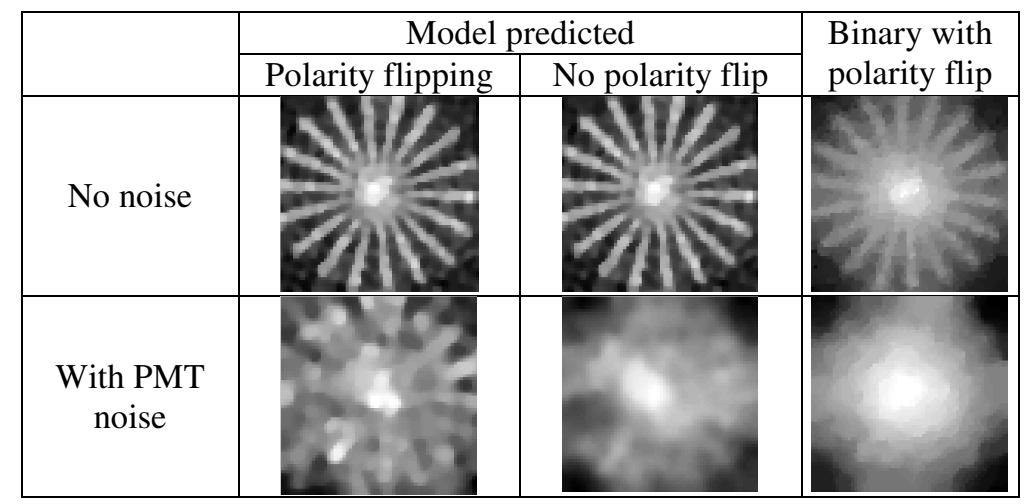

(a) Reconstructed images

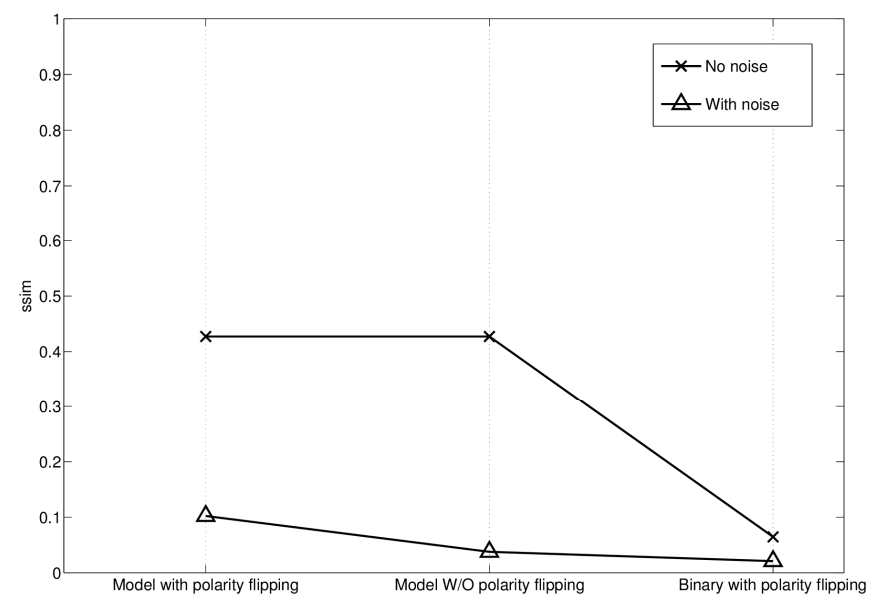

(b) Corresponding SSIM curves (X axis listed the three different cases).

Figure 12. Impact of different measurement matrix implementations with and without PMT noise.

Figures 13 and 14 demonstrate CS imager performance against various test images at different turbidities. These images consisted of both test patterns (Star Chart and Geometry patterns) and images taken in underwater environments (coral, fish, and diver). In Figure 14, the structural SSIM at various turbidities is presented. 


\begin{tabular}{|c|c|c|c|c|c|c|}
\hline \multirow{2}{*}{ Turbidities } & \multicolumn{6}{|c|}{ Images } \\
\hline & Star chart & Geometry & Coral 1 & Fish & Coral 2 & Diver \\
\hline \multicolumn{7}{|l|}{ Original } \\
\hline \multirow{2}{*}{\multicolumn{7}{|c|}{$\begin{array}{l}\text { Clear } \\
\text { water } \\
(0 \mathrm{AL})\end{array}$}} \\
\hline & & & & & & \\
\hline \multicolumn{7}{|l|}{$3 \mathrm{AL}$} \\
\hline \multicolumn{7}{|l|}{$5 \mathrm{AL}$} \\
\hline \multicolumn{7}{|l|}{$7 \mathrm{AL}$} \\
\hline
\end{tabular}

Figure13. Reconstruction images at different turbidities.

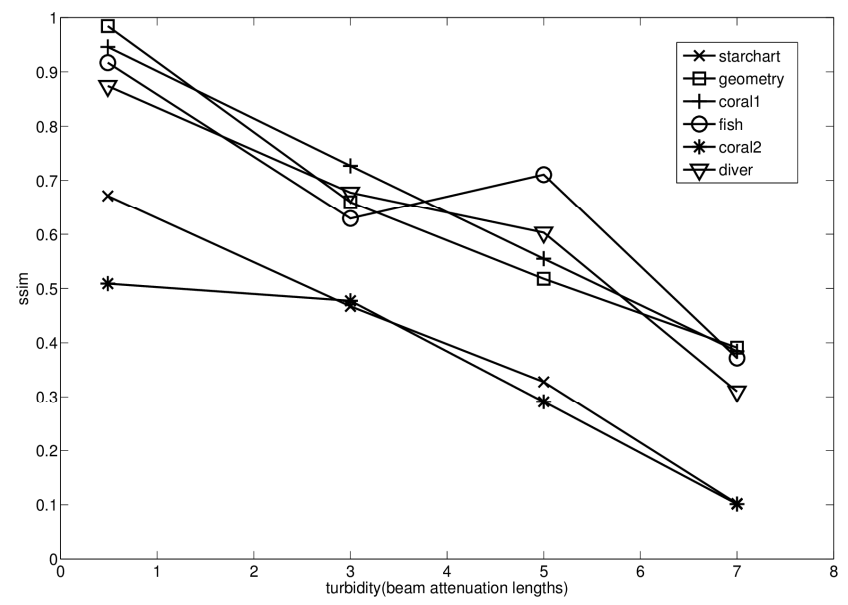

Figure14. Reconstruction Performance at different turbidities (SSIM metrics).

As seen from Figure 14, the quality degraded with increasing turbidity for all of the test images.

One interesting observation was that the SSIMs for the Star Chart and Coral 2 were lower than those for the other images at the corresponding turbidities. This was mainly due to the fact that the edges 
were sparser in the other patterns, which resulted in better performance of the TV minimization based optimization. At the range of $7 \mathrm{AL}$ all test images suffered significant loss of detail, which was due to the noise interfering with the reconstruction.

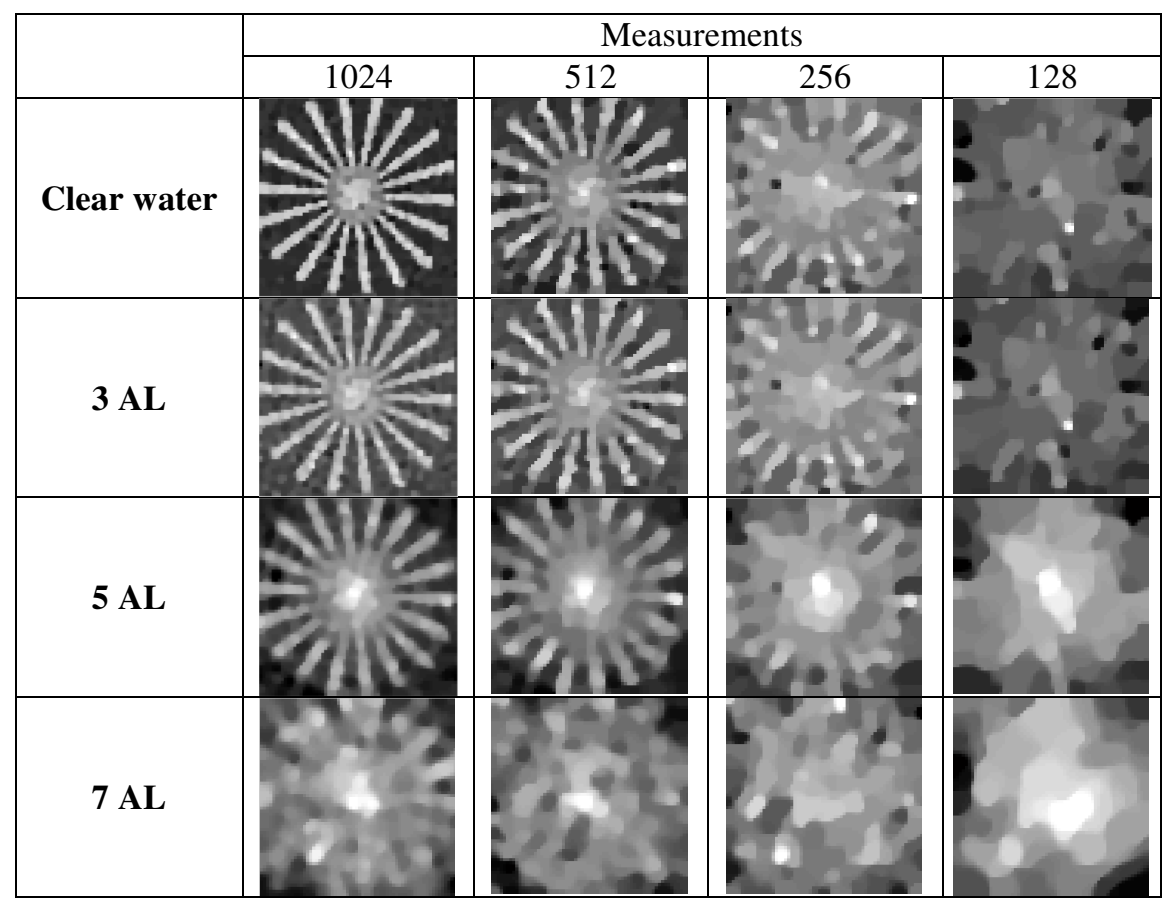

(a) Reconstructed images

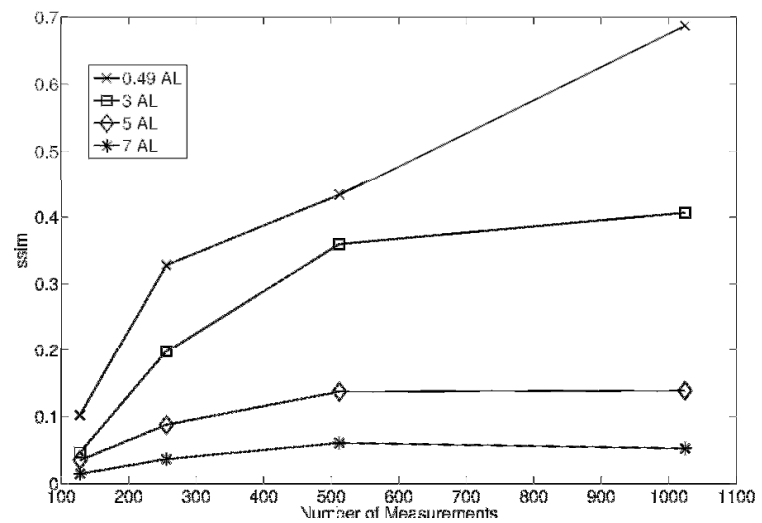

(b) Corresponding SSIM curves

Figure15. Reconstruction performance with different number of measurements.

Figure 15 illustrates the image quality change with different numbers of measurements (i.e., different compression ratios). It is interesting to note that there was more obvious image quality degradation with reduced measurements in clearer waters than at higher turbidities. 


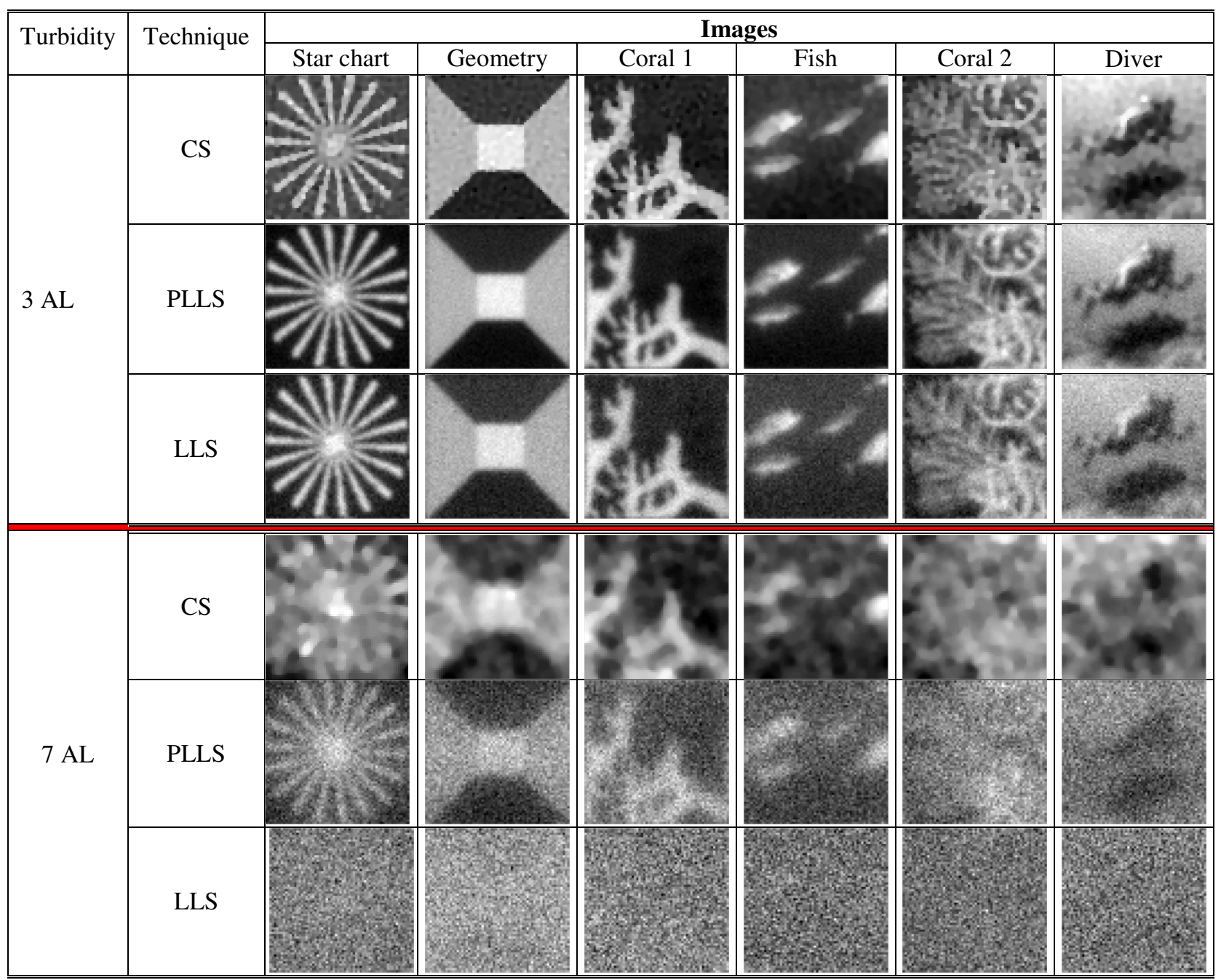

(a) Simulated images at different turbidities using the three different techniques
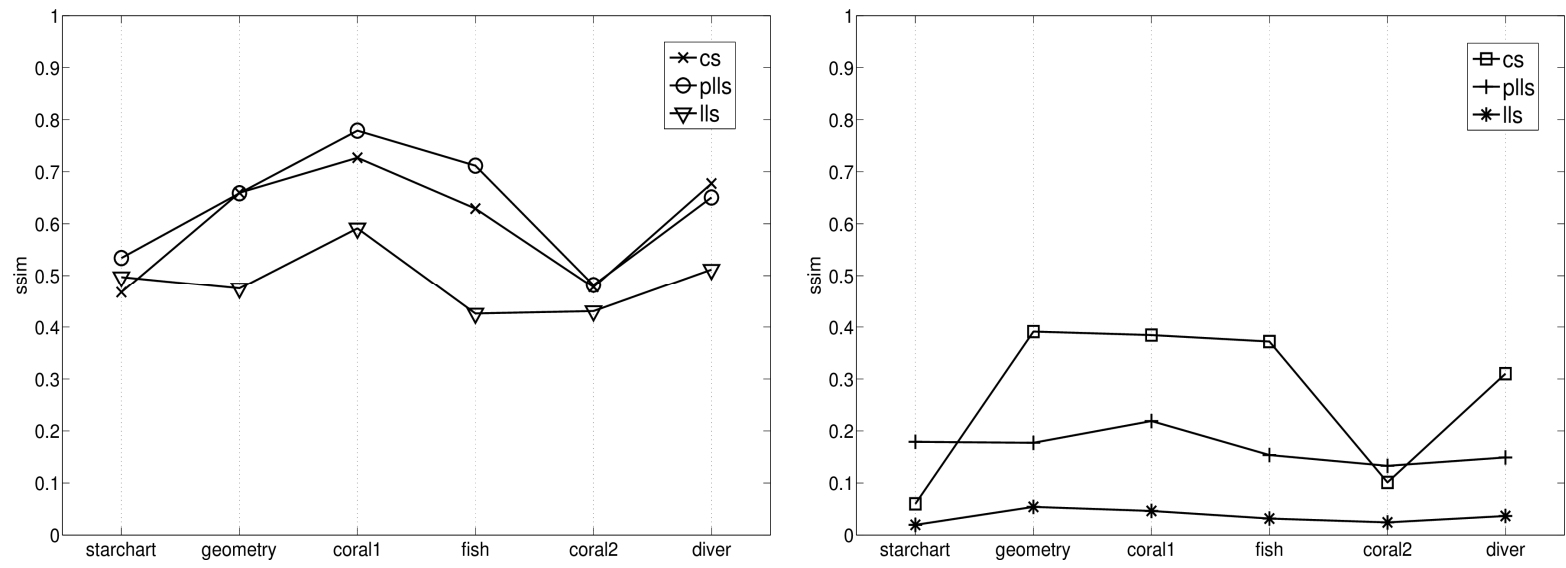

(b) SSIM Curves with turbidity at $3 \mathrm{AL}$

(c) SSIM curves with turbidity at $7 \mathrm{AL}$

Figure16. Reconstruction performance at different turbidities. 
The CS results were compared with the images from PLLS and CW LLS simulations in Figure 16. For the SSIM curves, the names of the test images are listed on the x-axis. It can be seen that the image quality of the CS imager was generally comparable to that of PLLS and LLS at lower turbidity (3 AL). At a higher turbidity (7 AL) the PLLS images preserved the image detail the best; CS images suffered more resolution loss than PLLS, especially for images with more high-resolution content; and, the CW LLS images were overwhelmed by noise. One reason for the relatively poor performance of the CS imager at high turbidity was that the current image reconstruction model (Eq. 4) assumed an additive Gaussian noise corruption, which deviated from the true noise characteristics of the PMT receiver. It is conceivable that a reconstruction model that better reflects the PMT noise could significantly improve CS imager performance, especially at higher turbidities.

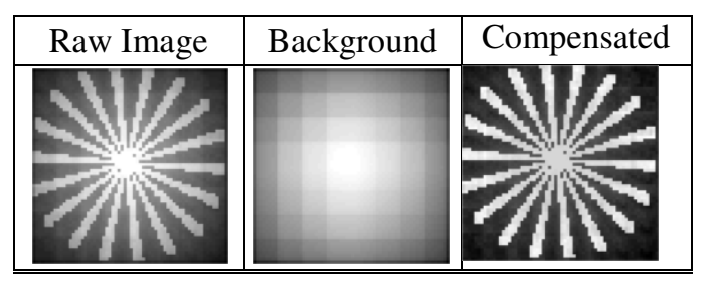

Figure 17. SIRGC at $3 \mathrm{AL}$ with no noise.

Finally, the CS imager was compared to the SIRGC. When there was no noise added, the results were in good agreement with those reported in [7] (Figure 17). Figure 18 shows the results when noise was added in the simulation. To improve the SNR in the SIRGC simulation, each sub-pattern was imaged 50 times and averaged. At the higher turbidity (7 AL), the SIRGC image was dominated by noise. This can be explained by observing that for both CS and SIRGC imagers, only a small fraction of energy reached/reflected from each target element; however, for the CS imager only the total reflected photons were used in image reconstruction, whereas with SIRGC, the camera needed to discern the intensity for each individual target element. 


\begin{tabular}{|c|c|c|c|}
\hline \multirow{2}{*}{ Technique } & \multicolumn{2}{|c|}{ Turbidities } & \\
\cline { 2 - 4 } $3 \mathrm{AL}$ & $\mathrm{CS}$ & PLLS & SIRGC \\
\hline & & & \\
& & & \\
& & & \\
$7 \mathrm{AL}$ & & & \\
& & & \\
\hline
\end{tabular}

(a) Simulated images using CS, PLLS and SIRGC

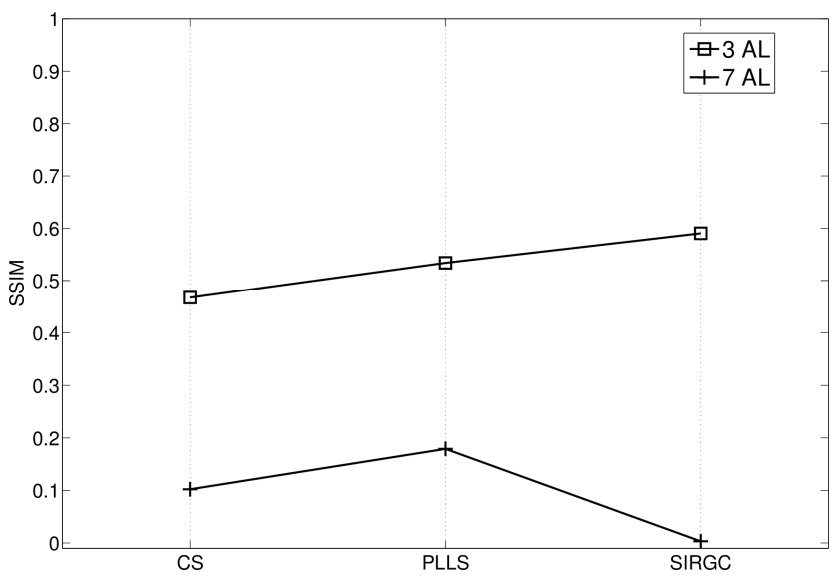

(b) Corresponding SSIM Curves at two different turbidities (X axis listed the three techniques)

Figure 18. Comparisons among CS, PLLS and SIRGC.

At 3 beam attenuation lengths, while the SIRGC image was noisy, it appeared to retain good image resolution. However, the SIRGC simulation results warrant some additional comments. First, in SIRGC, in addition to generating the illumination patterns, the DMD also serves as the receiver mask in front of the camera image plane. As in most confocal imaging applications, such a pinhole mask helps to achieve the balance of the light intensity and sharpness. By adopting the same singlepixel mask as in [7], the simulation assumed the smallest pinhole and therefore maximum sharpness; however, the impact on the noise performance due to the photon loss from such a small pinhole was not considered in the simulation. Second, adopting the PMT noise model in the simulation also helped to improve the noise performance since a PMT in general provides better SNR than the CCD 
devices in the camera. On the other hand, while in the simulation each sub pattern was imaged 50 times to improve the SNR of the SIRGC system, high-speed cameras capable of 1 million frame per second (FPS) are now commercially available. Therefore, a thorough study of the performance of SIRGC will need to weigh the tradeoffs among all of these factors. This, however, is beyond the scope of the present investigation.

\subsection{Experimental Results}

\subsubsection{Over-the-air Experimental Results}

An over-the-air experiment was conducted using a simple desktop setup. The measurement matrices were loaded onto a DELL M109S DLP pico-projector to illuminate the $10 \times 10 \mathrm{~cm}$ target placed $0.5 \mathrm{~m}$ away from the illuminator and receiver. 800 measurements were used to reconstruct images at a resolution of $64 \times 64$ pixels. Figure 19 shows the setup (a) and the reconstruction results for two technical targets (b and c).

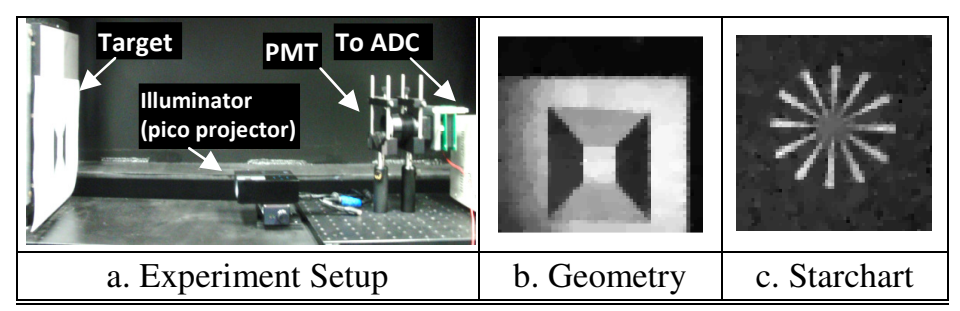

Figure 19. In-air experimental setup and results.

\subsubsection{Underwater Experimental Results}

Following the over-the-air experiment, an initial underwater experiment was conducted in the HBOI optical test tank to validate the proposed frame-based compressive sensing serial imaging design. The target plane consisted of multiple technical targets (Geometry and Star Chart) and coral samples (Figure 20a). A LabView based data acquisition system was developed for this effort. A series of pre-generated illumination pattern images were loaded onto a DELL 4310WX DLP data projector with a $1280 \times 800$ native resolution to project the patterns onto the target plane with a $60 \mathrm{~Hz}$ 
refresh rate. A Hamamatsu R7600U PMT was connected to a National Instrument data acquisition board to sample the PMT output (Figure 20b and c).

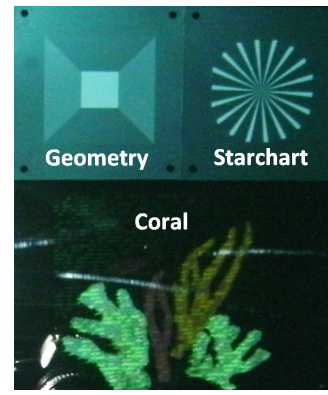

(a) Target plane

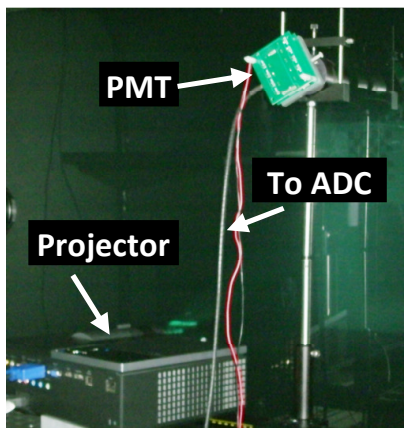

(b) System setup
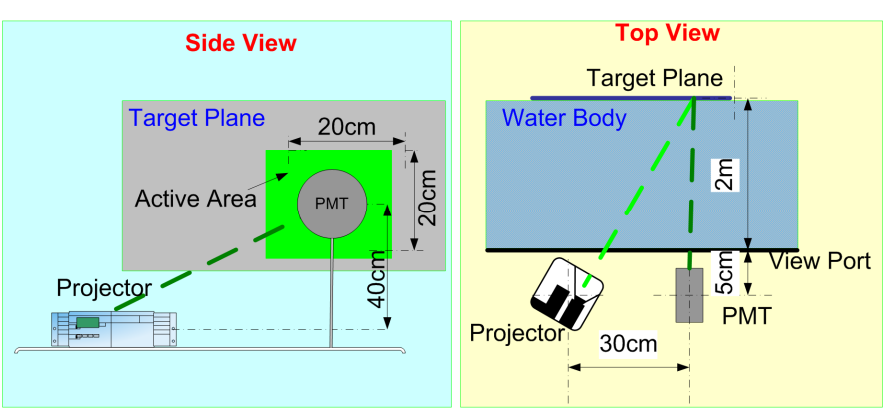

(c) Experimental environment

Figure 20. System setup geometry.

During the experiment, to achieve the necessary contrast for pattern projection, the projector was operated at a $20 \%$ brightness level with a light output of about 600 lumens. The dimensions of the measurement matrices were $100 \times 100$ pixels; the dimensions of the corresponding projected illumination patterns were $300 \times 300$ pixels, so that each element of the measurement matrix is covered by a $3 \times 3$ patch. With the distance between the screen and the projector set at $2 \mathrm{~m}$, the onscreen active area is about $20 \times 20 \mathrm{~cm}$. Such an area is suitable for both technical targets (with a dimension of $14 \times 14 \mathrm{~cm}$ ) and most of the coral targets.

As shown in Figure 20c, there is a $30 \mathrm{~cm}$ separation between the projector and the PMT. Since the optical arrangement of the projector is such that the light is projected with an upward tilt (optimized for conference room use), the elevation of the PMT is $40 \mathrm{~cm}$ higher than the projector so that the center of the illuminated area is aligned with the FOV of the PMT. A bandpass filter with $5 \mathrm{~nm}$ full width at half maximum (FWHM) centered at $532 \mathrm{~nm}$ was placed in front of the PMT to retain only the green portion of the spectrum. The PMT had a wide FOV of 13 degrees. The experiment was conducted at four different turbidities: clear water, $c=0.31,1$, and 2 . 


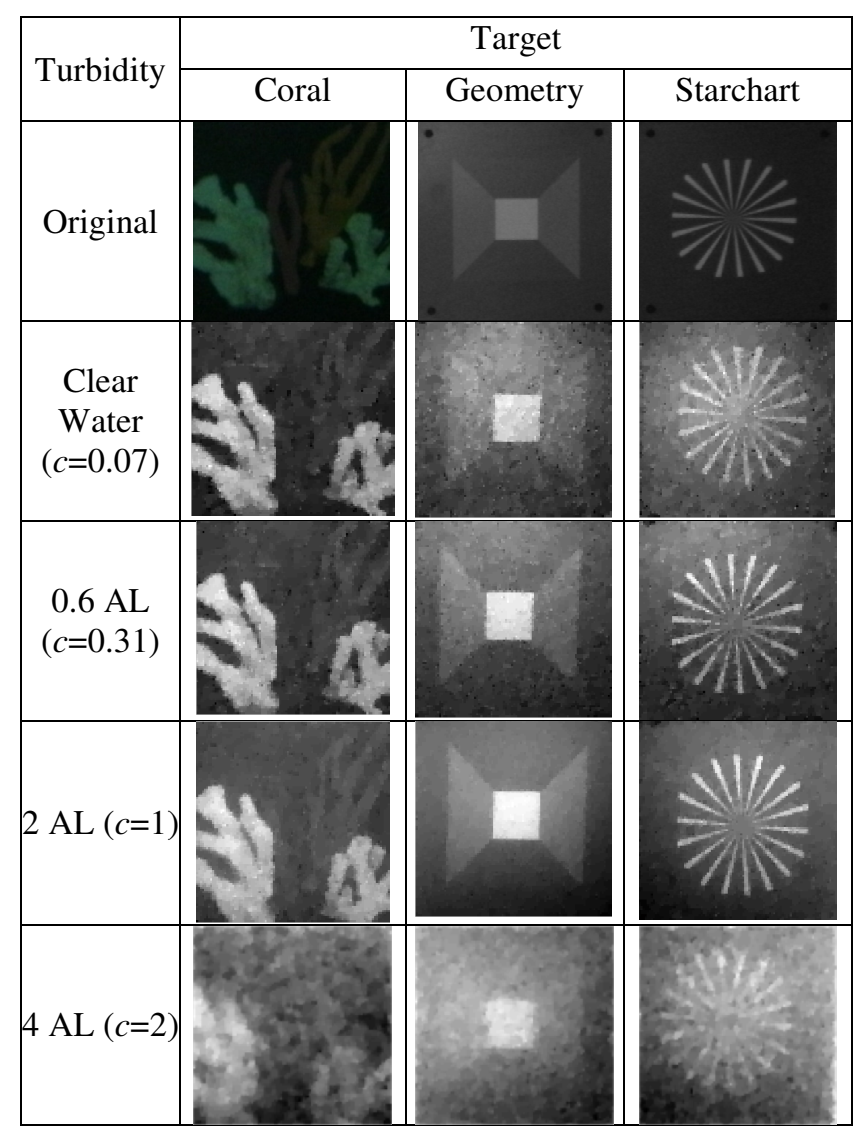

Figure 21. Reconstructed image at different turbidities.

Figure 21 illustrates the reconstructed images at different turbidities. All images are at a resolution of $100 \times 100$ pixels, and 2500 measurements were used in the reconstruction to give an effective compression ratio of $4: 1$. It can be observed that for the technical targets, the quality of the reconstructed images initially improves with increasing turbidity, though this trend breaks down at the highest turbidity of 4 beam attenuation lengths (due to the power limitation of the illuminator) . The main reason for the initial improvement was that the specular reflections from the surfaces of these two targets were reduced with increased turbidity. On the other hand, the coral results maintained fairly consistent quality at different turbidities, though this trend also broke down at 4 beam attenuation lengths. While the center portion of the corals is clearly identifiable in the color 
image, the coral reflectivity at $532 \mathrm{~nm}$ (green light) is extremely low, as can be observed in Figure 22, which results in a low-contrast image reconstruction of this region.

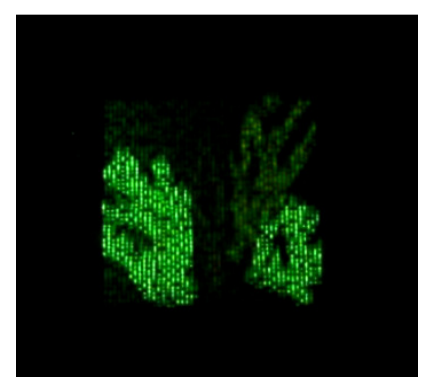

Figure 22. Reflection of the projected pattern from the corals.

In general, the current implementation of the proposed approach seems to favor higher contrast regions of the image (as can be seen with the Geometry target in clear water as well). Additional work will be required to address this issue. One advantage of the CS approach is that image reconstruction can begin when a minimum number of measurements are acquired, and image quality can then be progressively improved with additional measurements. Therefore, one potential approach is to dynamically identify the high-contrast and low-contrast regions from initial reconstructions, and adjust the intensity of the projected patterns to accentuate the low-contrast regions. One such attempt for the Geometry target is shown in Figure 23. To compensate for the high-contrast center block, half of the patterns were projected with the center square intentionally dimmed by $50 \%$, which helped to improve the results in the low-contrast regions.

\begin{tabular}{|c|c|c|}
\hline & Normal patterns & $\begin{array}{l}\text { Half normal patterns } \\
\text { and half dim patterns }\end{array}$ \\
\hline $\begin{array}{l}\text { Projected } \\
\text { Patterns }\end{array}$ & 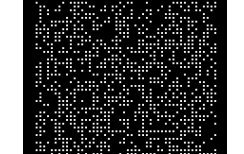 & 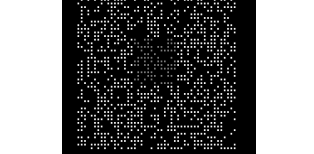 \\
\hline $\begin{array}{c}\text { Reconstructed } \\
\text { Image }\end{array}$ & & \\
\hline
\end{tabular}

Figure 23. Dynamical pattern adjustment for contrast improvement. 
The instruments used in the experiment were crude. The data projector, in particular, was not optimized for such experiments, and very low power was used to illuminate the target, with only a narrow part of the green spectrum reaching the PMT. Still, the results validated the proposed approach.

\section{SUMMARY}

The proposed compressive sensing (CS) based laser serial imager is an attempt to extend CS theory to the field of imaging through scattering media, specifically in the underwater environment. While the proposed technique is still at an early stage of the development, the simulations and experimental results presented above lend credibility to the basic design concept. The proposed design has the potential to improve system reliability and compactness, and to reduce system costs over the current state-of-the-art laser line scan imagers. On the other hand, since the CS imager inherits the highly photon efficient "bucket" light collector (PMT) used in the LLS system; it may offer better photon efficiency (hence better noise performance) than SIRGC in higher turbidities.

Model-assisted CS is an attractive concept since it can help extend CS to other applications involving scattering media, such as fog, mist, smoke, or dust. In addition to improving the image acquisition rate by reducing the required measurements via the "compression-while-sampling" CS paradigm, another important implication is the possibility of operating the sampling electronics at lower frequency, hence improving the system noise performance. On the other hand, while range gating was not employed in the simulations/experiments conducted for this study, the proposed CS technique can be applied in conjunction with the pulsed laser and range-gating to reduce volume backscattering that overlaps with the reflected target signal (albeit at the expense of high frequency 
electronics). This trade-off will be an interesting and important research topic, which will help to gain a better understanding of the underwater CS imaging system.

Another important future research topic is the development of a reconstruction model that incorporates the well-studied PMT noise characteristics better. In this regard, investigating the PMT noise model in [27] as well as the Poisson CS reconstruction technique proposed in [32] and [33] will be beneficial. Some other research topics include the tradeoff between DMD refresh rate and number of measurements (i.e., the rate-distortion curve) at different turbidities, adaptive sensing patterns to improve the low-contrast region in the presence of high-contrast objects, more efficient measurement matrix design and the effects of the environmental and system variations (i.e., system excursion/motion, ocean wave/turbulence etc.).

To rigorously study the aforementioned topics and further improve the proposed technique requires the development of both a simulation environment that truly reflects the CS underwater imager (the current environment was developed based on a radiative transfer model for the LLS imager) and prototype systems for experimental work in both the test tank and in the field.

It is worth mentioning that the work reported here relates to a frame-based implementation, where the measurements of the overall target scene are acquired simultaneously and are, therefore, more compatible with hover-capable AUVs or ROVs. We are also interested in developing a CS imaging system that is more compatible with traditional underwater near mono-static electro-optical system designs, where the image is formed in a line-by-line fashion, relying on the vehicle's forward motion to complete a 2D image. The Distributed Compressive Video Sensing (DCVS) concept [28, 29], which uses the correlation between adjacent video frames to reduce the required number of measurements, is worthy of investigation for such a system concept. In this context, each line can be 
regarded as a video frame, and the correlations/redundancy between adjacent lines will be exploited via DCVS to improve the overall compression performance.

Finally, Table 1 presents a comparison of CS with two other aforementioned configurations: PLLS and SIRGC.

Table 1. Comparison of Three Underwater Laser Imaging Techniques.

\begin{tabular}{|c|c|c|c|}
\hline & CS & SIRGC & PLLS \\
\hline System Design & $\begin{array}{l}\text { Using Solid State SLM (DMD) } \\
\text { to modulate laser and PMT; } \\
\text { Compressing during sampling; }\end{array}$ & $\begin{array}{l}\text { Using Solid State SLM (DMD) } \\
\text { to modulate laser and high } \\
\text { sensitivity camera based } \\
\text { receiver; } \\
\text { Compressing after sampling; } \\
\end{array}$ & $\begin{array}{l}\text { Mechanical actuator and high } \\
\text { precision prism to direct laser } \\
\text { and PMT; } \\
\text { Compressing after sampling; }\end{array}$ \\
\hline $\begin{array}{l}\text { Imaging } \\
\text { Method and } \\
\text { Speed }\end{array}$ & $\begin{array}{l}\text { Serial Imaging, actual speed } \\
\text { depends on compression ratio } \\
\text { Additional speed advantage for } \\
\text { video. }\end{array}$ & $\begin{array}{l}\text { Parallel Imaging, Should be } \\
\text { fastest among all three. }\end{array}$ & $\begin{array}{l}\text { Serial Imaging. Maybe slowest } \\
\text { among all three. Scanner speed } \\
\text { is limiting factor. }\end{array}$ \\
\hline $\begin{array}{c}\text { Imager } \\
\text { Configuration }\end{array}$ & $\begin{array}{l}\text { Can be setup with near mono- } \\
\text { static or bi-static configuration. } \\
\text { Not require line-of-sight from } \\
\text { target to PMT }\end{array}$ & $\begin{array}{l}\text { Requires direct line-of-sight } \\
\text { from target to camera; not be } \\
\text { suitable in bi-static } \\
\text { configuration. Optical alignment } \\
\text { could be challenging. }\end{array}$ & $\begin{array}{l}\text { Can be setup with near mono- } \\
\text { static or bi-static configuration. } \\
\text { Requires line-of-sight from } \\
\text { target to PMT }\end{array}$ \\
\hline $\begin{array}{c}\text { Photon } \\
\text { Efficiency } \\
\text { /Concentration }\end{array}$ & $\begin{array}{l}\text { Less than LLS due to the } \\
\text { photon loss in SLM, but at } \\
\text { same level. } \\
\text { Photon/pixel depends on } \\
\text { compression ratio. }\end{array}$ & $\begin{array}{l}\text { Power shared among all active } \\
\text { pulses - comprise with imaging } \\
\text { speed. Faster speed = lower } \\
\text { photon Concentration. }\end{array}$ & $\begin{array}{l}\text { Highest, all incident power used } \\
\text { to construct pixel intensity }\end{array}$ \\
\hline $\begin{array}{l}\text { Motion } \\
\text { Artifacts }\end{array}$ & $\begin{array}{l}\text { Platform excursion can be } \\
\text { modeled as Gaussian noise in } \\
\text { measurements, handled as } \\
\text { constraints in reconstruction. }\end{array}$ & $\begin{array}{l}\text { Misalignments of consecutive } \\
\text { SLM pulses induces artifacts. } \\
\text { Can be addressed in image } \\
\text { synthesis; }\end{array}$ & $\begin{array}{l}\text { Pixel misalignment due to } \\
\text { motion. Can be compensated } \\
\text { with known platform motion } \\
\text { data or deconvolutoin. }\end{array}$ \\
\hline $\begin{array}{c}\text { Image } \\
\text { Reconstruction }\end{array}$ & $\begin{array}{l}\text { Computationally intensive } \\
\text { optimization required. }\end{array}$ & $\begin{array}{l}\text { Image synthesis comparative low } \\
\text { intensity. }\end{array}$ & $\begin{array}{l}\text { Minimum additional process } \\
\text { required. }\end{array}$ \\
\hline
\end{tabular}

\section{ACKNOWLEDGEMENTS}

This work was conducted under a grant monitored by the US Office of Naval Research and institutional funding from Harbor Branch Oceanographic Institution at Florida Atlantic University. The authors would like to thank Mr. David Rashkin for helping with the PMT noise model. The authors also want to thank HBOI for providing the underwater imagery used in the simulations. 


\section{REFERENCES}

[1] F. R. Dalgleish and F. M. Caimi, "Synchronous Laser Line Scanners for Undersea Imaging Applications," Accepted as a book chapter for Taylor and Francis series in Optical Engineering (Laser and Optical Scanning).

[2] J.S. Jaffe, "Computer Modeling and the Design of Optimal Underwater Imaging Systems", IEEE J of Ocean Engineering 15, 101-111 (1990).

[3] T.J. Kulp, D. Garvis, R. Kennedy, T. Salmon, Cooper, K. "Results of the final tank test of the LLNL/NAVSEA Synchronous-Scanning Underwater Laser Imaging System", Ocean Optics XI, SPIE proceedings, Vol. 1750, pp. 453-464. 1992.

[4] G. R. Fournier, D. Bonnier, J. Luc Forand, and P. W. Pace, "Range-gated underwater laser imaging system", Opt. Eng. 32, 2185. 1993.

[5] S. Q. Duntley, R. W. Austin, R. L. Ensminger, T. J. Petzold, and R. C. Smith, "Experimental TVI System Report,” Visib. Lab. Tech. Rep., pp. 74-1, 1974.

[6] F. R. Dalgleish, B. Ouyang, A. K. Vuorenkoski, B. Metzger, B. Ramos and W R. Britton, “Extended range distributed laser serial imaging in turbid estuarine and coastal conditions," Proc. MTS/IEEE Oceans'12, 2012.

[7] J. S. Jaffe, "Extended Range Optical Imaging using One and Two Dimensional Structured Illumination,” Optics Express, Vol. 18, pp. 12328-12340, 2010.

[8] D. Donoho, “Compressive Sensing,” IEEE Trans. Inform. Theory, vol. 52, pp. 1289-1306, 2006.

[9] E. Candes and T. Tao, "Near Optimal Signal Recovery from Random Projections: Universal Encoding Strategies,” IEEE Trans Inform. Theory, vol. 52, pp. 5406-5425, 2006.

[10] S. Chen, D. Donoho, M. Saunders, “Atomic Decomposition by Basis Pursuit,” SIAM J. Science Comp., 20, pp. 33-61, 1999. 
[11] E. Candes and J. Romberg, "Sparsity and Incoherence in Compressive Sampling," Inverse Problems, vol. 23, pp. 969-985, 2007.

[12] L. Gan, T. Do and T. Tran. "Fast Compressive Imaging using Scrambled Block Hadamard Ensemble". Proc. EUSIPCO, 2008.

[13] M. Duarte, M. Davenport, D. Takhar, J. Laska, T. Sun, K. Kelly and R. Baraniuk, "Single-Pixel Imaging via Compressive Sensing," IEEE Signal Processing Magazine, vol. 25, pp. 83-91, 2008.

[14] R.G. Baraniuk, “Compressive Sensing,” IEEE Signal Processing Magazine, vol. 24, pp. 118121, 2007.

[15] T. Do, T. Tran, and L. Gan, "Fast Compressive Sampling with Structurally Random Matrices," Proc. ICASSP, pp. 3369 - 3372, 2008.

[16] E. Candes and J. Romberg, "Robust Signal Recovery from Incomplete Observations," Proc. ICIP, pp. 1281-1284, 2006.

[17] E. Candes, J. Romberg, and T. Tao, "Stable Signal Recovery from Incomplete and Inaccurate Measurements," Comm. Pure Appl. Math., vol. 59, pp. 1207-1223, 2006.

[18] E. Candes, J. Romberg, and T. Tao, "Robust Uncertainty Principles: Exact Signal Reconstruction from Highly Incomplete Frequency Information," IEEE Trans. Inform. Theory, vol. 52, pp. 489-509, 2006.

[19] E. Candes and J. Romberg, "11-magic: Recovery of Sparse Signals via Convex Programming", http://users.ece.gatech.edu/ justin/11 magic/, 2005.

[20] D. Dudley, W. Duncan and J. Slaughter, "Emerging Digital Micromirror Device (DMD) Applications," Proc of SPIE, 2003, 4985, 14-25.

[21] M. Lustig, D. Donoho, and J. M. Pauly, "Sparse MRI: The Application of Compressed Sensing for Rapid MR Imaging,” Magnetic Resonance in Medicine, vol. 58, pp. 1182 - 1195, 2007. 
[22] J. Ma, “Single-Pixel Remote Sensing," IEEE Geoscience and Remote Sensing Letters, Vol. 6, pp. $199-203,2009$.

[23] R. G. Baraniuk and T. P. H. Steeghs, "Compressive Radar Imaging," IEEE Radar Conference, 2007.

[24] T. E. Giddings and J. J. Shirron, "Numerical Simulation of the Incoherent Electro-optical Imaging Process in Plane-Stratified Media," Opt. Eng. vol. 48, no. 12, 126001 (2009).

[25] M. F. Duarte, M. B. Wakin, D. Baron, and R. G. Baraniuk, "Universal distributed sensing via random projections", Proc. Information Processing in Sensor Networks (IPSN), 2006.

[26] Y. Rachlin, and D. Baron, "The secrecy of compressed sensing measurements" , Proc. Allerton Conf. on Communication, Control, and Computing, 2008.

[27] B. Ouyang, F. R. Dalgleish, A. Vuorenkoski, W. Britton, B. Ramos and B. Metzger, "Visualization for Multi-static Underwater LLS System using Image Based Rendering", IEEE Journal of Oceanic Engineering (Accepted).

[28] M. Stojanovic, "Underwater Acoustic Communications," in Encyclopedia of Electrical and Electronics Engineering, John G. Webster, Ed., John Wiley \& Sons, 1999, Vol.22, pp.688-698. [29] T. E. Giddings, "Photomultiplier Receiver Model for Electro-Optical Systems”, Metron Tech. Rep., 2008.

[30] D. Rashkin, I. Cardei, M. Cardei F. R. Dalgleish T. E. Giddings, "Detector Noise Model Verification For Undersea Free Space Optical Data Links”, Proc. MTS/IEEE Oceans'12, 2012. [31] Z. Wang, A. C. Bovik, H. R. Sheikh and E. P. Simoncelli, "Image quality assessment: From error visibility to structural similarity," IEEE Transactions on Image Processing, vol. 13, no. 4, pp. 600-612, Apr. 2004. 
[32] Z. Harmany, R. Marcia, and R. Willett, "Sparse Poisson Intensity Reconstruction Algorithms", IEEE Workshop on Statistical Signal Processing, 2009.

[33] V. Studer, J. Bobin, M. Chahid, H. Moussavi, E. J. Cand'es, and M. Dahan, “Compressive Fuorescence Microscopy for Biological and Hyperspectral Imaging", Proc. of the National Academy of Sciences USA, vol. 109, no. 26, pp. E1679-E1687, 2012.

[34] T. T. Do, Y. Chen, D. T. Nguyen, N. Nguyen, L. Gan, and T. D. Tran, "Distributed compressed video sensing", Proc. IEEE Int. Conf. on Image Processing, 1393-1396, 2009.

[35] L. W. Kang and C. S. Lui, "Distributed compressive video sensing," Proc. IEEE Int. Conf. on Acoustics, Speech and Signal Processing (ICASSP 09), pp. 1169-1172, 2009. 